D. Cuisance ${ }^{1}$

D. Demba ${ }^{2}$

B. Vallat ${ }^{3}$

A. Kota-Guinza ${ }^{2}$

F. D'Amico ${ }^{4}$

F. Ndokoué 2

\title{
Répartition des glossines dans la zone d'action agropastorale de Yérémo en République centrafricaine
}

CUISANCE (D.), DEMBA (D.), VALLAT (B.), KOTA-GUINZA (A.), D'AMICO (F.), NDOKOUE (F.). Répartition des glossines dans la zone d'action agropastorale de Yérémo en République centrafricaine. Revue Élev. Méd. vét. Pays trop., 1994, 47 (1) : $69-75$

Sous l'effet de divers facteurs, le cheptel zébu Mbororo de la RCA s'est considérablement accru durant ces dernières années $(2 \quad 200000$ têtes) du fait des potentialités pastorales offertes. En vue d'une meilleure gestion de cet espace, des zones d'activité agropastorale ont été créées (ZAGROP). En contrepartie du disponible fourrager, cet élevage en zone humide doit affronter des contraintes pathologiques accrues, dominées notamment par les trypanosomoses. Pour éviter que l'usage massif actuel des trypanocides par les éleveurs n'aboutisse à une impasse, l'Agence Nationale de Développement de l'Elevage (ANDE) a demandé d'identifier des méthodes de lutte contre les glossines. Dans l'une de ces ZAGROP (60 $000 \mathrm{ha}$ ), la première étape a consisté à élablir une carte précise de la distribution des espèces vectrices grâce à un piégeage systématique (piège biconique). Glossina fuscipes fuscipes (10 805 glossines capturées) occupe la totalité du réseau hydrographique avec des densités faibles à moyennes ( 3 glossines par piège et par jour). Glossina fusca congolensis est rare (7 individus capturées) et Glossina morsitans submorsitans, autrefois présente, semble avoir disparu. Les éléments sur l'abondance, la diversité et la distribution des espèces sont discutés. Ils servent de base au choix d'une méthode de lutte par piégeage actuellement mise en oeuvre dans ce milieu pastoral.

Mots-clés : Bovin - Elevage - Glossina fuscipes fuscipes - Glossina fusca congolensis - Glossina morsitans submorsitans - Distribution Lutte anti-insecte - Piège - Zone humide - République centrafrique.

\section{INTRODUCTION}

A partir de 1986 sont créées en RCA des zones d'activité agropastorale (ou ZAGROP) dans lesquelles l'Agence Nationale de Développement de l'Elevage confie à des éleveurs des lieux de pâturage en leur demandant en contrepartie un effort de gestion (rotation des pâturages, mises en défens, contrôle des feux, etc.) avec l'appui de structures d'encadrement et la participation de la Fédération Nationale des Eleveurs Centrafricains (17). Un des thèmes techniques retenus est l'identification de méthodes de lutte contre les glossines, à la fois efficaces mais également autogérables par les éleveurs (9). En effet, le problème des trypanosomoses animales se pose

1. CIRAD-EMVT c/o Centre ORSTOM, BP 5045, 34032 Montpellier cedex, France.

2. ANDE, BP 1500, Bangui, République centrafricaine.

3. Ministère de la Coopération, sous-direction du Développement économique et de l'Environnement, 1 bis Avenue de Villars, 75007 Paris, France.

4. Parc national des Pyrénées, Cyabas, 64440 Laruns, France.

Reçu le 1.10.1993, accepté le 14.12.1993. avec acuité pour ce bétail zébu Mbororo qui a "glissé" progressivement du plateau de Bouar et du plateau de l'Adamaoua camerounais ainsi que d'autres pays voisins (Tchad en particulier) vers le centre et l'est centrafricain, passant de zones sans ou avec peu de glossines vers des zones d'infestation permanente.

Les ventes de trypanocides et de trypanopréventifs (plus de 1500000 doses annuelles) sont révélatrices de l'importance de cette pathologie et des pertes occasionnées sur un cheptel de plus de 2 millions de bovins dans ce pays $(18,20)$. La ZAGROP de Yérémo est l'une des premières zones "pilotes" créées et, dans ce contexte, les premiers essais de lutte contre les glossines sont mis en place. Ils sont précédés par une évaluation de la situation entomologique pendant 7,5 mois de prospection, pour dresser la carte précise de distribution des glossines, étape préliminaire à toute campagne de lutte. Elle permet de connaître le nombre d'espèces ou de sous-espèces de glossines présentes, leur importance relative (densité apparente) et leur occupation spatiale en vue d'identifier l'espèce-cible et les possibilités d'intervention.

\section{DESCRIPTION DE LA ZONE}

D'une superficie de 62000 ha, la ZAGROP de Yérémo est à cheval sur l'axe routier Bouar-Bangui (160 km de la capitale), très près de Bossembélé (carte 1). Le climat est de type soudano-guinéen (sous-climat de type soudanooubanguien) avec une pluviométrie de 1400 à $1600 \mathrm{~mm}$ et un indice d'Aubreville 7-3-2. La température moyenne annuelle est de $24^{\circ} 3 \mathrm{C}$. Cette zone se situe dans le domaine phytogéographique soudano-guinéen, secteur sud (Daniellia oliveri, Terminalia glaucescens), district occidental des plateaux de Bossembélé à Cussonia djalonensis et Hannoa undulata (4). Les savanes arbustives et arborées constituent les parcours naturels de cette zone exploités sur un mode extensif. Elles sont traversées par un réseau dense d'environ $750 \mathrm{~km}$ de rivières, généralement permanentes et bordées par des galeries forestières. Ces dernières sont de largeur variable (5 à $100 \mathrm{~m}$ environ). Hautes et denses, elles sont souvent fermées au-dessus du lit encaissé des rivières.

En 1987, la ZAGROP de Yérémo abritait environ 20000 têtes de bétail appartenant à 240 éleveurs dispersés sur l'ensemble de la zone. Quelques petits villages d'agricul- 




Carte 1 : Plan de situation de la ZAGROP de Yérémo (adapté du Grand Atlas de Géographie, Encyclopedia Universalis, 1986).

teurs jalonnent la route nationale qui la traverse. La faune sauvage, autrefois abondante, a très fortement régressé et n'est plus représentée que par des suidés sauvages (phacochères, potomachères), des petites antilopes (céphalophes et guibs), des reptiles (varans, crocodiles), et quelques rares grands mammifères (cobes de Buffon, buffles, hippopotames).

\section{MATÉRIEL ET MÉTHODE}

A l'échelle nationale, la carte de répartition générale des glossines établie en 1963 par FINELLE et al. (13) indique à cette époque la présence de trois espèces près de Bossembélé, Glossina fuscipes fuscipes (glossine ripicole), Glossina morsitans submorsitans (glossine savanicole) et Glossina fusca congolensis (glossine forestière). D'après ces renseignements de base, ainsi que les informations des éleveurs et quelques sondages préalables, la prospection est dirigée sur le réseau de galeries forestières et secondairement sur les savanes.

\section{Galeries forestières}

Trois équipes techniques, composées chacune de 4 personnes, sont munies de 25 pièges biconiques $(5,6)$. $\mathrm{Ce}$ piège est retenu, car il est considéré comme le plus performant pour les glossines ripicoles et permet une comparaison avec des données obtenues ailleurs (Côted'Ivoire, Mali, Burkina Faso, etc.). Aucun attractif olfactif n'est utilisé car peu ou pas de produits efficaces ne sont connus vis-à-vis de ces espèces de glossines d'Afrique centrale.

A chaque équipe est associé un éleveur ou l'un de ses fils, celui-ci séjournant habituellement sur le secteur prospecté. Cette participation permet d'intéresser l'éleveur aux vecteurs des trypanosomoses mais aussi de profiter de sa bonne connaissance du terrain (accès des galeries, emplacement des abreuvoirs à bétail, zones de forte densité en insectes, présence de faune, etc.). Chaque équipe prospecte de façon méthodique la partie du réseau hydrographique qui lui est confiée chaque jour par le responsable en mettant en place les pièges au bord de 
TABLEAU I Bilan de la prospection entomologique dans la Zone d'Action Agropastorale de Yérémo (RCA) (décembre 1987 à juillet 1988).

\begin{tabular}{|c|c|c|c|c|c|c|c|c|c|}
\hline & & \multirow{2}{*}{$\begin{array}{l}\text { Nombre } \\
\text { pièges }\end{array}$} & \multicolumn{2}{|c|}{$\begin{array}{l}\text { G. fuscipes } \\
\text { fuscipes }\end{array}$} & \multicolumn{2}{|c|}{$\begin{array}{l}\text { G. fusca } \\
\text { congolensis }\end{array}$} & \multicolumn{2}{|c|}{$\begin{array}{l}\text { G. morsitans } \\
\text { submorsitans }\end{array}$} & \multirow{2}{*}{$\begin{array}{c}\text { Glossine/ } \\
\text { piège/ } \\
\text { jour }\end{array}$} \\
\hline & & & $M$ & $F$ & $M$ & $F$ & $M$ & $F$ & \\
\hline $\begin{array}{l}\text { Partie Nord } \\
\text { Galeries forestières }\end{array}$ & $\begin{array}{l}\text { La Doumi Lin } \\
\text { Bou } \\
\text { Bi }\end{array}$ & $\begin{array}{l}903 \\
728 \\
483\end{array}$ & $\begin{array}{r}1721 \\
956 \\
621\end{array}$ & $\begin{array}{r}1883 \\
1149 \\
697\end{array}$ & $\begin{array}{l}0 \\
0 \\
0\end{array}$ & $\begin{array}{l}0 \\
0 \\
0\end{array}$ & $\begin{array}{l}0 \\
0 \\
0\end{array}$ & $\begin{array}{l}0 \\
0 \\
0\end{array}$ & $\begin{array}{c}\text { Moyenne } \\
3,32\end{array}$ \\
\hline \multirow[t]{2}{*}{ Savane } & & 77 & 1 & 4 & 0 & 0 & 0 & 0 & \\
\hline & $\mathrm{S} / \mathrm{T}$ & 2191 & \multicolumn{2}{|c|}{7032} & 0 & 0 & 0 & 0 & \\
\hline $\begin{array}{l}\text { Partie Sud } \\
\text { Galeries forestières }\end{array}$ & $\begin{array}{l}\text { La Doumi } \\
\text { Mbakin } \\
\text { Mbombo } \\
\text { Mbi } \\
\text { Bo } \\
\text { Bi }\end{array}$ & $\begin{array}{r}117 \\
107 \\
155 \\
77 \\
469 \\
145\end{array}$ & $\begin{array}{r}138 \\
126 \\
147 \\
50 \\
1216 \\
33\end{array}$ & $\begin{array}{r}275 \\
123 \\
177 \\
99 \\
1332 \\
47\end{array}$ & $\begin{array}{l}0 \\
3 \\
0 \\
0 \\
0 \\
0\end{array}$ & $\begin{array}{l}2 \\
0 \\
1 \\
0 \\
1 \\
0\end{array}$ & $\begin{array}{l}0 \\
0 \\
0 \\
0 \\
0 \\
0\end{array}$ & $\begin{array}{l}0 \\
0 \\
0 \\
0 \\
0 \\
0\end{array}$ & $\begin{array}{c}\text { Moyenne } \\
3,51\end{array}$ \\
\hline \multirow[t]{2}{*}{ Savane } & & 34 & 3 & 7 & 0 & 0 & 0 & 0 & \\
\hline & $\mathrm{S} / \mathrm{T}$ & 1104 & $\begin{array}{r}1713 \\
3\end{array}$ & $3^{2060}$ & 3 & 4 & 0 & 0 & \\
\hline \multicolumn{2}{|c|}{$\mathrm{T}$} & 3295 & \multicolumn{2}{|c|}{10805} & \multicolumn{2}{|c|}{7} & \multicolumn{2}{|c|}{0} & \\
\hline
\end{tabular}

l'eau à raison de 1 piège tous les $200 \mathrm{~m}$, de préférence à un endroit ouvert et ensoleillé. Selon les difficultés d'accès, chaque équipe pose de 15 à 25 pièges par jour entre $8 \mathrm{~h}$ et $10 \mathrm{~h} 30$ et les retire entre $15 \mathrm{~h} 30-18 \mathrm{~h}$ en progressant donc de 3 à $5 \mathrm{~km}$ par jour, soit 6 à $10 \mathrm{~km}$ parcourus au total chaque jour.

\section{Savanes}

En savane, les transects sont faits en fonction de la présence des pistes d'accès et sont installés dans les principales parties de la zone à raison de 1 piège tous les kilomètres, posé en bordure des pistes et laissé en place pendant la même durée que précédemment. L'emploi d'un véhicule roulant à très faible vitesse pour la pose des pièges constitue un moyen supplémentaire de détecter l'espèce savanicole, bien connue pour pénétrer dans les véhicules.

En fin de journée, les glossines sont triées après identification par espèce et par sexe et les résultats sont portés sur le fond cartographique au 1/50 000 dressé à partir des images satellitaires SPOT du 15 novembre 1986 et du 25 décembre 1987 réalisées pour les besoins d'un autre projet (DE WISPELAERE, communication personnelie). Chaque équipe possède un jeu de cartes. La présence de résidus sanguins dans le tube digestif de cer- tains individus permet en outre l'identification ultérieure de l'hôte nourricier de la glossine. Pour des raisons d'accès, l'enquête s'est déroulée essentiellement en saison sèche, au début et en fin de saison des pluies entre juin 1987 et juillet 1988, soit pendant 7,5 mois de travail effectif.

\section{RESULTATS (tableau I, carte 2)}

Sur ce réseau de $750 \mathrm{~km}$ de galeries forestières, $650 \mathrm{~km}$ sont prospectés, soit 85 p. 100. La longueur des galeries forestières est plus grande au nord $(430 \mathrm{~km})$ qu'au sud $(220 \mathrm{~km})$, de l'axe routier (RN 3). 3295 pièges sont posés au cours de 7,5 mois de prospection, dont 3184 le long des galeries forestières et 111 en savane. Les captures ont été les suivantes pendant cette période :

\section{- 10805 Glossina fuscipes fuscipes}

- 7 Glossina fusca congolensis

\section{- 0 Glossina morsitans submorsitans}

G. m. submorsitans apparaît absente dans la zone prospectée avec le moyen de capture utilisé pour la période considérée ; cette absence est corroborée par les dires des éleveurs qui ne signalent pas de glossines en savane tout au long de l'année. 
D. Cuisance D. Demba B. Vallat A. Kota-Guinza F. D'Amico F. Ndokoué

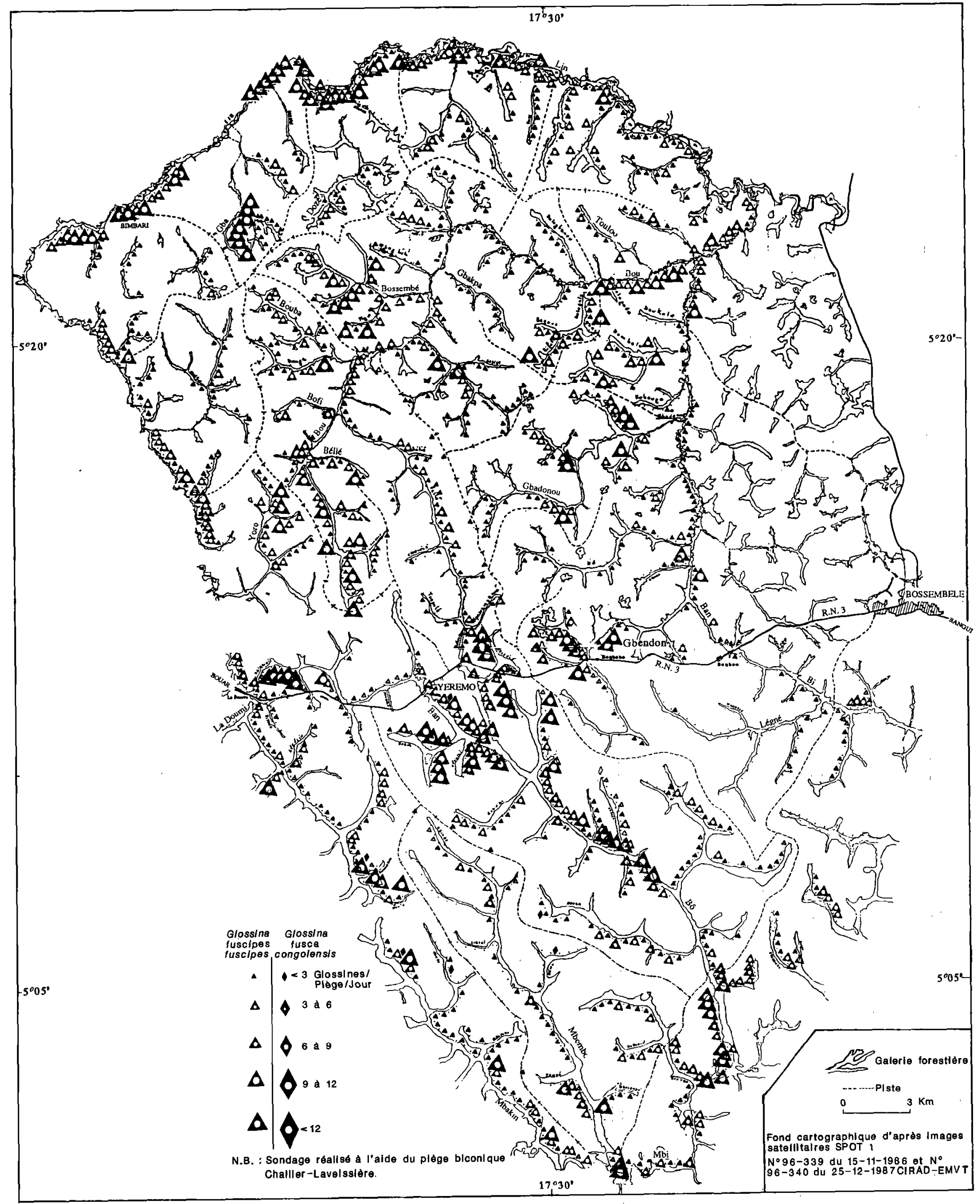

Carte 2 : Carte de répartition des glossines dans la zone d'action agropastorale de Yérémo. 
G. $f$. fuscipes est la sous-espèce dominante. Sa densité apparente exprimée en glossines/piège/jour est en moyenne de 3,32 pour la partie de la ZAGROP au nord de la route nationale et de 3,51 pour la partie au sud. Les densités sont donc faibles à moyennes sur l'ensemble de la zone, mais l'examen de la carte de distribution montre qu'elles sont très hétérogènes pouvant devenir fortes à très fortes en certains points. Ceux-ci sont presque toujours les lieux fréquentés par le bétail ou la faune (abreuvoirs) ou par l'homme (lavoirs, points de pêche, gués, ponts, points de rouissage du manioc, etc.). En mettant en parallèle une carte de recensement des campements peuls établie à la même période (M. BEREKOUTOU, communication personnelle), il apparaît une forte concordance avec la situation entomologique. G. $f$. fuscipes, glossine essentiellement riveraine, est capable de faire des incursions en savane en saison humide comme le montrent les captures de quelques exemplaires sur des pistes de ligne de crête passant à plusieurs centaines de mètres des galeries forestières les plus proches, ces individus isolés n'étant pas ou peu repérés par les éleveurs.

G. fusca congolensis est présente mais en très faible densité. Celle-ci est probablement sous-estimée, car cette glossine est faiblement attirée et capturée par ce type de piège. De plus, si elle est active toute la journée en saison des pluies, les maxima se situeraient plutôt en fin d'après-midi en saison sèche, donc au moment du retrait des pièges. Ses incursions en savane sont rares, de faible amplitude et de courte durée $(22,23)$. Quelques Tabanidés et stomoxes sont récoltés mais en faible nombre dans cette zone pendant la durée de la prospection.

\section{DISCUSSION}

La comparaison de la situation entomologique actuelle avec celle décrite par FINELLE et al. (1963), à 25 ans d'écart, n'est pas facile, car les méthodes d'échantillonnage sont différentes : captures par pièges avec sondage intensif systématique d'un côté et captures manuelles avec sondages ponctuels au filet de l'autre. Toutefois, il semble que G. $m$. submorsitans ait disparu, du moins pendant la période de prospection puisqu'elle n'est ni vue, ni capturée, que ce soit par les pièges ou par les véhicules réputés très attractifs pour cette glossine. L'absence absolue de cette sous-espèce est toujours difficile à prouver du fait de la faible efficacité des moyens de capture (attractivité réduite de l'homme ou des pièges existants) mais aussi de sa grande capacité, tant à envahir saisonnièrement les savanes qu'à s'en retirer (12). Cette mobilité peut induire une appréciation erronée de la situation.

Dans cette zone, l'enquête entomologique de 7,5 mois a été suivie d'un contrôle périodique mensuel par capture avec pièges au niveau de 10 points sélectionnés ( 2 pièges par point pendant 4 jours consécutifs) pendant un an, sans qu'aucune G. m. submorsitans soit trouvée. Le recul de cette glossine savanicole peut être attribuable à la raréfaction, par braconnage, d'une faune de mammifères sauvages qui constitue ses hôtes préférés (12), mais cette explication n'est que partiellement vraie, car des zones voisines et de situation cynégétique comparable sont actuellement envahies par $G$. $m$. submorsitans et le bétail semble avoir pris le relais de la faune pour expliquer cette "explosion" (plateau de l'Adamaoua au Nord-Cameroun, zone de Samorogouan au Burkina Faso).

G. fusca congolensis est toujours présente mais demeure rare puisque 7 individus sont capturés, uniquement dans la partie sud de la zone dans le bassin de la rivière Mbi (affluents Mbakin, Mbombo, Bô). Les galeries forestières de ce système hydrographique, proche de la zone forestière, sont en effet beaucoup plus larges et denses que dans la partie nord. Cet excellent vecteur des trypanosomoses animales (13) est probablement légèrement sousestimé dans cette enquête du fait du moyen de capture et des moments de mise en place (milieu de journée), mais dans cette zone, il reste un vecteur insignifiant du fait de sa très faible représentation. En revanche, G. f. fuscipes est omniprésente sur l'ensemble du réseau hydrographique de cette zone agropastorale. Son grand opportunisme alimentaire (21) lui permet de vivre aux dépens d'hôtes aussi variés que reptiles, mammifères domestiques et sauvages ainsi que l'homme (16).

Toutefois, l'élévation de densité enregistrée au niveau des abreuvoirs à bétail $(2,9)$ et l'accroissement des proportions de repas de sang sur bovins pendant la saison de sédentarité (mai à novembre) (CUISANCE, non publié, GOUTEUX et al., (16)) laissent supposer que cette glossine est le vecteur essentiel des trypanosomoses animales dans cette zone. Une étude épidémiologique approfondie est actuellement en cours dans une autre zone d'élevage, comparant les prévalences trypanosomiennes chez le bétail et chez $G$. $f$. fuscipes en relation avec l'écologie de cette sous-espèce et des vecteurs mécaniques potentiels.

Les Tabanidés et les stomoxes sont en faible nombre, mais les résultats des captures ne reflètent pas la réalité car ces insectes fréquentent davantage les aires de repos du bétail $(10,11)$ autour du campement que les galeries forestières. De plus, l'efficacité de ce piège est réduite vis-à-vis de certaines espèces (en cours d'évaluation). Le moyen de sondage utilisé (piège biconique placé pendant 6 à 7 heures) et l'intensité du sondage (1 piège tous les $200 \mathrm{~m}$ ) rendent ce type de prospection assez lourd à gérer mais permettent en contrepartie d'avoir une meilleure image de la situation avant de choisir une stratégie de lutte. Celle-ci sera en effet fort différente selon que le vecteur à contrôler ou à éliminer est $G$. $f$. fuscipes et/ou G.m. submorsitans. Si seule G. f. fuscipes est soupçonnée et si l'objectif visé est de contrôler les densités, une enquête allégée est acceptable (2). En revanche, pour $G$. $m$. submorsitans, il est impérieux de s'assurer de l'exacte distribution de cette redoutable glos- 


\section{Cuisance D. Demba B. Vallat A. Kota-Guinza F. D'Amico F. Ndokoué}

sine et si possible à chaque saison. Les résultats malheureux de campagnes précipitées sans connaissance précise de la situation initiale sont là pour rappeler la nécessité d'une prospection méthodique couvrant toute la surface à assainir.

La participation des éleveurs ou de leurs enfants à la prospection faite par les équipes techniques offre la possibilité d'une sensibilisation et d'une initiation de ces communautés aux vecteurs des trypanosomes par apprentissage in situ (démonstration de pose des pièges et de récoltes d'insectes) tout en permettant aux techniciens de recueillir de précieuses indications de terrain (accès aux galeries, présence de faune, densité d'insectes, importance locale de la pathologie, etc.).

\section{CONCLUSION}

Cette phase de prospection de la ZAGROP de Yérémo a permis de réactualiser la distribution générale des glossines dans cette région en montrant la disparition de $G$. $m$. submorsitans dont la limite sud se trouve maintenant plus au nord. Elle a surtout permis de dresser un état précis de la distribution et de la densité apparente des trois espèces présentes (échelle 1/50 000) en montrant que $G$. $f$. fuscipes, seule glossine omniprésente et dominante, constitue probablement le vecteur essentiel des trypanosomes du bétail zébu dans cette zone. Contre cette glossine, qui occupe presque tout le réseau hydrographique, des expérimentations récentes ont permis d'identifier un piège simple, peu coûteux et efficace (14, 15). La distribution linéaire de cette espèce et sa tendance à l'agrégation au niveau de certains lieux, en particulier les abreuvoirs à bétail, sont les éléments retenus pour la mise en oeuvre d'une campagne originale de lutte par piégeage ponctuel qui est actuellement entreprise $(1,3,8,15,19)$.

Cet ensemble de données entomologiques géographiquement référencées doit permettre le croisement avec des cartes de végétation (issues des images satellitaires) ainsi qu'avec des données sur l'occupation spatiale des troupeaux pour la constitution d'un système d'information géographique.

\section{BIBLIOGRAPHIE}

1. BLANC (F.). Lutte anti-glossinaire en République centrafricaine. Mémoire ESAT-1 Montpellier, 1991, 118 p. + annexes.

2. BLANC, (F.), GOUTEUX (J.P.), CUISANCE (D.), POUNÉKROZOU (E.), N'DOKOUE (F.), LE GALL (F.). Etude de la répartition des tsé-tsé (Diptera : Glossinidae) en zone de savane humide (République centrafricaine). Evaluation de techniques de prospection entomologique. Trop. Med. Parasit., 1991, 42: 127-130.

3. BLANC (F.), GOUTEUX (J.P.), CUISANCE (D.), POUNÉKROZZUU (E.), LE MASSON (A.), N'DOKOUE (F.), MAINGUET (M.), D'AMICO (F.), LE GALL (F.). La lutte par piégeage contre Glossina fuscipes fus- cipes pour la protection de l'élevage en République centrafricaine. III. Vulgarisation en milieu Mbororo. Revue Elev. Méd. vét. Pays trop., 1991, 44 (3) : 301-307

4. BOULVERT (Y.). Carte phytogéographique à $1 / 1000000$, République centrafricaine. Paris, ORSTOM, 1986.131 p. (Notice explicative $\mathrm{n}^{\circ} 104$ )

5. CHALLIER (A.), LAVEISSIÈRE (C.). Un nouveau piège pour la capture des glossines. Description et essais sur le terrain. Cah. ORSTOM sér. Ent. méd. Parasit. ,1973 11: 251-262.

6. CHALLIER (A.), EYRAUD (M.), LAFAYE (A.), LAVEISSIÈRE (C.) Amélioration du rendement du piège biconique pour glossines (Diptera, Glossinidae) par l'emploi d'un cône inférieur bleu. Cah. ORSTOM, sér. Ent. méd. Parasit., 1977, 15 : 283-286.

7. CUISANCE (D.). La lutte contre les glossines dans la zone d'action agro-pastorale de Yérémo. Bilan de quatre missions d'appui à l'unité de lulte contre les glossines dans le cadre du Projet National de Développement de l'élevage de la R.C.A. RCA, Ministère du Développement rural Direction générale de l'Elevage et des Industries animales, Bangui. Maisons-Alfort, CIRAD-EMVT, $1988.66 \mathrm{p}$.

8. CUISANCE (D), CAILTON (P), KOTA-GUINZA (A.), N'DOKOUÉ (F.), POUNÉKROUZOU (E.), DEMBA (D.). Lutte contre Glossina fúscipes fuscipes par piégeage chez les éleveurs Mbororo de République centrafricaine. Revue Elev. Méd. vét. Pays trop., 1991, 44 (1) : 81-89.

9. CUISANCE (D.), GOUTEUX (J.P.), CALLTON (P.), KOTA-GUINZA (A.), N'DOKOUÉ (F.), POUNÉKROUZOU (E.), DEMBA (D.) Problématique d'une lutte contre les glossines pour la protection de l'élevage zébu en RCA. Mém. Soc. r. belge Ent., 1992, 35 : 103-110.

10. D'AMICO (F.). Rôle de Glossina fuscipes fuscipes Newstead 1910 dans la transmission des trypanosomoses bovines en. Afrique Centrale. Le cas de la zone d'élevage d'Ouro-Djafoun (République centrafricaine). Thèse Doct. ès Sciences, Université Montpellier II, 1993. 160 p. + annexes.

11. D'AMICO (F.) MOUSSA-POUSSINGA (J.), GOUTEUX (J.P.), LE GALL (F.), BLANC (F.), MAINGUET (M.), GUILLERME (J.M.) KOTA-GUINZA (A.), CUISANCE (D.). Les Stomoxyinae dans une zone d'élevage en savanes humides de République $c$ entrafricaine. Leur rôle dans la transmission des trypanosomoses bovines In : 14e Réunion du Groupe Ad hoc de l'OIE sur les trypanosomoses animales non transmises par les glossines, Paris, 1993.

12. DIALLO (A) Glossina morsitans submorsitans Newstead, 1910 (Dip tera : Clossinidae). Son ćcologic ct son rôle dans les trypanosomoses animales en zone de savane soudano-guinéenne du Mali (Ranch de. MadinaDiassa). Thèse Doct. es Sciences Naturelles, Aix-Marseille III,' 1985.149 p.

13. FINELLE (P.), ITARD(J.), YVORE (P.), LACOTTE (R.). Répartition des glossines en RCA : état actuel des connaissances. Revue Élev. Méd. vét. Pays trop., 1963, 16 (3) : 337-348.

14. GOUTEUX (J.P), CUISANCE (D), DEMBA (D), N'DOKOUÉ (F) LE GALL (F.). La lutte par piégeage contre Glossina fuscipes fuscipes pour la protection de l'élevage en République centrafricaine. I. Mise au point d'un piège adapté à un milieu d'éleveurs semi-nomades. Revue Elev. Méd. vét. Pays trop., 1991, 44 (3) : 287-294.

15. GOUTEUX (J.P.), LE GALL (F.). Piège bipyramidal à tsé-tsé pour la protection de 1'élevage en République centrafricaine. Revue mond. Zootech., $1992(1-2): 37-43$.

16. GOUTEUX (J.P.), D'AMICO (F.), CUISANCE (D), BLANC (F) DEMBA (D.), STAAK (C.), CLAUSEN (P.H.), KOTA-GUINZA (A.), LE GALL (F.). Les hôtes de Glossina fuscipes fuscipes Newstead, 1910 (Diptera: Glossinidae) dans deux zones d'élevage de la République centrafricaine. Vet. Res., 1994, 25 : 16-28. 
17. KOTA-GUINZA (A.), LE MASSON (A.). Un essai de gestion de l'espace en République centrafricaine. Les zones d'action agropastorale (ZAGROP). Possibilités et contraintes. In : Actes du séminaire régional sur les fourrages et l'alimentation des ruminants, Ngaoundéré (Cameroun), 1620 novembre 1987. Tome 1. Maisons-Alfort, IEMVT, Yaoundé, IRZ 1990. p. 49-68. (Etudes et Synthèses de l'IEMVT n ${ }^{\circ}$ )

18. LE GALL (F.), N'DOKOUÉ (F.), MAINGUET (M.). Résultats d'une enquête large réalisée sur 27 secteurs d'élevage en RCA : maladies transmises par les tiques et trypanosomoses. Espèces vectrices, coûts des moitalités et traitements. Bangui, RCA, Rapport DSARA/ANDE, 1992. 53 p.

19. LE GALL (F.), BLANC (F.), GOUTEUX (J.P.), MAINGUET (M.), CUISANCE (D.), LEMESRE (J.L.), NITCHEMAN (S.), CAVALEYRA (M.), D'AMICO (F.), POUNÉKROUZOU (E.), N’DOKOUÉ (F.). La lutte par piégeage contre Glossina fuscipes fuscipes pour la protection de l'élevage en République centrafricaine. IV - Impact entomologique, parasitologique et zootechnique (soumis pour publication)

CUISANCE (D.), DEMBA (D.), VALLAT (B.), KOTA-GUINZA (A.) D'AMICO (F.), NDOKOUE (F.). Tsetse fly distribution in agropastoral areas of Yérémo in Central African Republic. Revue Élev. Méd. vét. Pays trop., 1994, 47 (1) : 69-75

The number of Mbororo zebu cattle in Central African Republic has considerably increased the last few years $(2,200,000$ heads $)$ due to various factors such as new pastoral potentialities. With a view to improving the management of these herds and spaces, agropastoral activity areas (ZAGROP) were created.In compensation with the forage potentialities, the rearing of livestock in humid areas is subject to enhanced pathological constraints mainly caused by trypanosomosis. To prevent problems related to the present massive use of trypanocidal drugs by the cattle owners, the National Agency for Development of Livestock Production (ANDE) has started to identify the control methods applied against tsetse flies. In one of these agropastoral areas $(60,000$ ha), the first step was to establish an accurate map of vector species distribution using a systematic trapping method. Glossina fuscipes fuscipes (10,805 trapped tsetse flies) was found throughout the whole hydrographic system with a low to moderate density (3 tsetse flies/trap/day). Glossina fusca congolensis was seldom caught ( 7 trapped individuals) and Glossina morsitan submorsitans, which was formerly present, seemed to have disappeared. Data on species abundance, diversity and distribution are discussed. They are used as a basis for choosing a control method by trapping, presently set up in this pastoral area.

Key words : Cattle - Animal husbandry - Glossina fuscipes fuscipes Glossina fusca congolensis - Glossina morsitans submorsitans - Distribution - Insect control - Trap - Humid area - Central African Republic.
20. LE MASSON (A.), REMAYEKO (A.). Les éleveurs Mbororo. Etude socio-économique. Bangui (RCA), ANDE, 1990. 227 p.

21. VAN VEGTEN (J.A.). The tsetse fly Glossina fuscipes fuscipes 1911 in East Africa ; some aspects of its biology and its role in the epidemiology of human and animal trypanosomiasis. Thèse Doct. Université d'Amsterdam, 1971.132 p

22. YVORÉ (P.). Quelques observations sur l'écologie de deux glossines du groupe fusca en République centrafricainc. $I n$ : Actes de la $9^{\circ}$ réunion de l'ISCTR, Conakry, Guinée, 2l-25 août 1962. Londres, CCTA, 1963. p. 197-204. (publ. ISCTR n ${ }^{\circ} 88$ )

23. YVORÉ (P.), LACOTTE (R.), FINELLE (P.). Etude de la biologie et de l'écologie de Glossina fusca congolensis Newstead and Evans en République centrafricaine. I. Influence du climat et de la végétation sur la répartition et la densité des glossines. Revue Elev. Méd. vét. Pays trop., 1965, 18 (2) : 151-164

CUISANCE (D), DEMBA (D), VALLAT (B), KOTA-GUINZA (A.), D'AMICO (F.), NDOKOUÉ (F.). Distribución de las glosinas en la zona de acción agropecuaria de Yeremo en República centroafricana. Revue Élev. Méd. vét. Pays trop., 1994, 47 (1) : 69-75

A causa de varios factores, especialmente las potencialidades presentadas por los pastos, se ha aumentado considerablemente el ganado cebú Mbororo de la RCA durante los últimos años (2 200000 cabezas). Para un mejor manejo de dichos pastos, se crearon zonas de actividad agropecuaria (ZAGROP). A pesar de la ventaja de la disponibilidad del forraje esta ganadería tiene que arrostrar factores patológicos importantes dominados en particular por las tripanosomosis. Para evitar que el uso masivo actual de los tripanocidas por los ganaderos sea un estancamiento, la Gestoría Nacional de Desarrollo de la Ganadería (ANDE) pidió que se identifiquen los métodos de lucha contra las glosinas. En una de estas ZAGROP, el primer trabajo fué el establecimiento a una mapa precisa de la distribución de las especies vectores gracias de una captura sistemática con trampa bicónica. Demostró que Glossina fuscipes fuscipes (10 805 glosinas capturadas) ocupa la totalidad de la red hidrográfica con densidades reducidas a medias ( 3 glosinas/trampa/día). Glossina fusca congolensis es escasa (7 moscas capturadas) y Glossina morsitans submorsitans, en otro tiempo presente, parece ser desaparecida. Se discuten los elementos en lo relativo a la abundencia, la diversidad y la distribución de las especies. Sirven de base para la elección de un método de lucha mediante captura por trampa actualmente utilizado en este medio agropecuario.

Palabras clave: Bovino - Ganadería - Glossina fuscipes fuscipes - Glossina fusca congolensis - Glossina morsitans submorsitans - Distribución - Lucha contra los insectos - Trampa - Zona humeda - República centroafricana. 